\title{
Surfaces
}

\section{SCIENCES : QUI EST L’AUTEUR ?}

\section{Isabelle Stengers}

Volume 2, 1992

URI : https://id.erudit.org/iderudit/1065236ar

DOI : https://doi.org/10.7202/1065236ar

Aller au sommaire du numéro

\section{Éditeur(s)}

Les Presses de l’Université de Montréal

ISSN

1188-2492 (imprimé)

1200-5320 (numérique)

Découvrir la revue

Citer cet article

Stengers, I. (1992). SCIENCES : QUI EST L'AUTEUR ? Surfaces, 2.

https://doi.org/10.7202/1065236ar

\section{Résumé de l'article}

Une étude de l'aspect « littéraire » de la légitimation scientifique portant plus précisément sur les problèmes de l'invention, de la découverte et l'auteur comme modes de discours. d'utilisation que vous pouvez consulter en ligne.

https://apropos.erudit.org/fr/usagers/politique-dutilisation/ 


\title{
SCIENCES:
}

\section{QUI EST L'AUTEUR?}

\author{
$\underline{\text { Isabelle Stengers }}$
}

\section{RÉSUMÉ}

Une étude de l'aspect <<littéraire>> de la légitimation scientifique portant plus précisément sur les problèmes de l'invention, de la découverte et l'auteur comme modes de discours.

\begin{abstract}
A study of the textual aspects of scientific legimitation, with particular attention to the problems of innovation, authorship and discovery as modes of discourse.
\end{abstract}

\section{Prologue}

Au cours de la troisième journée du Discours concernant deux sciences nouvelles, Galilée, sous le masque de Salviati, énonce une définition du mouvement uniformément accéléré dont il prétend qu'elle rejoint l'essence du mouvement naturellement accéléré des corps graves: <<Je dis qu'un 
mouvement est également ou uniformément accéléré quand, partant du repos, il reçoit des moments égaux de vitesse $>>$. Pour nous, cette définition est le premier énoncé relevant de la physique moderne, le premier qui ait résisté au temps pour tracer à travers plus de trois siècles et demi le fil d'une continuité. Comment vont réagir à ce qui, pour nous, est un événement les interlocuteurs que Galilée a donnés à Salviati, Sagredo et Simplicio?

C'est Sagredo qui parle: $<<$ Bien que je n'aie rien, rationnellement parlant, contre cette définition-ci ou contre une autre, quel qu'en soit l'auteur, puisqu'elles sont toutes arbitraires, je puis cependant douter, soit dit sans vous offenser, qu'une telle définition, élaborée et acceptée dans l'abstrait, s'adapte et convienne au type de mouvement accéléré auquel obéissent les graves en tombant naturellement $>>$.

Sagredo est l'homme de bon sens, celui avec qui les lecteurs de Galilée doivent s'identifier. Stratégie d'une efficacité redoutable d'ailleurs, car lorsque Sagredo, oubliant son impartialité supposée, s'allie avec Salviati, dans le Dialogue concernant les deux grands système du monde, pour couvrir d'insultes le malheureux Simplicio et avec lui tous les auteurs adversaires de Galilée qu'il représente en vrac, ce sont les lecteurs qui sont, en même temps que lui, entraînés à commettre un véritable assassinat moral. Cette vérité de type nouveau inventée par Galilée, et qu'on appelle depuis vérité scientifique, s'annonce ouvertement dans le Dialogue comme une vérité de combat, se vérifiant à sa capacité de faire taire ou de ridiculiser ceux qui la contestent.

Mais dans les Discours, le ton a changé. Galilée a été condamné. Vieillard, il sait que sa mort est proche. Il écrit clandestinement pour des lecteurs qu'il ne connaîtra pas. Il écrit pour l'avenir. Simplicio et Sagredo sont devenus de simples faire-valoir, posant les questions et opposant les objections dont Galilée pense que c'est justement la force et la nouveauté de sa vérité que de pouvoir y répondre. Et l'objection de Sagredo annonce la principale épreuve que la vérité galiléenne aura à surmonter: vaincre le scepticisme avec lequel le bon sens accueille toute définition, celle-ci comme une autre, quel qu'en soit l'auteur. La qualité de l'auteur, son autorité ou la rationalité de ses arguments ne sont pas une garantie. Toute définition est élaborée dans l'abstrait. Toute définition est arbitraire. Toute définition, dirons-nous, est une fiction renvoyant à un auteur.

Sagredo représente le sens commun. Mais son objection traduit le fait que le sens commun n'est pas une donnée transhistorique. Car à l'époque de Galilée, l'argument de Sagredo était également l'argument du Pouvoir, en l'occurrence de l'Église romaine. Monseigneur Oreggi, devenu théologien personnel du pape Urbain VIII, nous a laissé le souvenir de l'entretien que 
celui-ci, alors cardinal Maffeo Barberini, eut avec Galilée après la première condamnation de 1616: <<Il lui demanda s'il était hors de la puissance et de la sagesse de Dieu de disposer et de mouvoir d'une autre manière les orbes et les astres, et cela, cependant, de telle sorte que tous les phénomènes qui se manifestent dans les cieux, que tout ce que l'on enseigne touchant le mouvement des astres, leur ordre, leur situation, leurs distances, leur disposition, puissent néanmoins être sauvés. Si vous voulez déclarer que Dieu ne pourrait le faire, il vous faut démontrer, ajouta le saint prélat, que tout cela ne pourrait, sans impliquer contradiction, être obtenu par un système autre que celui que vous avez conçu; Dieu peut, en effet, tout ce qui n'implique pas contradiction.[1]>> Le grand savant, conclut Monseigneur Oreggi, garda le silence.

Qu'Urbain VIII, retrouvant son argument dans la bouche de Simplicio à la fin du Dialogue, ait considéré que Galilée entendait ainsi le ridiculiser, puisque tout ce que dit Simplicio est par définition ridicule, appartient à la légendaire histoire de la condamnation de Galilée, sur laquelle je ne m'attarderai pas. L'argument, en revanche, m'intéresse parce qu'il brise la mise en scène élaborée par Galilée lui-même, et trop souvent reprise par ceux qui cherchent à caractériser la singularité des sciences dites modernes. Les adversaires de Galilée ne furent pas seulement les héritiers attardés d'Aristote, ce qui aurait pour effet de mettre entre parenthèses le Moyen-Age. La vérité qu'annonce Galilée n'a pas seulement à s'imposer contre une autre vérité qu'elle contredirait. Elle doit d'abord et avant tout s'imposer contre l'idée que toute connaissance générale est essentiellement une fiction, et qu'il n'appartient pas au pouvoir de la raison humaine de rejoindre la raison des choses, que celle-ci renvoie à l'ordre des causalités aristotéliciennes ou à la mathématique.

Barberini, futur Urbain VIII, évoque la toute puissance de Dieu: $<<$ Dieu peut tout ce qui n'implique pas contradiction $>>$. Il reprend, ce faisant, le célèbre argument d'Étienne Tempier, évêque de Paris, qui condamna en 1277 l'ensemble des thèses cosmologiques issues de la doctrine aristotélicienne. Fut ainsi condamnée la proposition selon laquelle $<<$ Dieu ne pourrait imprimer au Ciel un mouvement de translation, car ce mouvement produirait un vide dont l'existence ne peut être admise sans absurdité $>>$. L'absurdité n'est pas la contradiction. L'absurdité renvoie à l'idée d'une rationalité qui établirait, d'une manière ou d'une autre, un trait commun entre la raison humaine et les choses, de telle sorte que l'argumentation rationnelle soit en mesure de revendiquer le pouvoir de faire la différence entre le possible et l'impossible, le convenable et l'inconvenant, le pensable et l'inconcevable. C'est ce trait commun que vient réfuter la référence à la toute puissance du divin auteur de la création. Si Dieu l'avait voulu, ce qui nous semble normal ne le serait pas, ce qui nous semble inconcevable ou miraculeux serait la norme. La toute puissance de Dieu impose que nous pensions sur fond de risque, que nous osions par exemple, comme le fit Samuel Butler dans Erewhon, penser qu'une société aurait pu exister où la maladie et la malchance seraient sévèrement punis, 
alors que les crimes et les délits entraîneraient la pitié et les soins médicaux les plus attentifs.

$\mathrm{Si}$, entre les mondes fictifs imaginables et notre monde, aucune autre différence ne peut être légitimement invoquée que la seule volonté de Dieu, tout mode de connaissance qui ne se réduit pas soit à la logique, soit au pur constat, est de l'ordre de la fiction plus ou moins bien construite. Chaque fiction peut avoir ses avantages, mais toutes renvoient à un auteur défini d'abord et avant tout par son incapacité de remonter aux raisons de la décision divine de créer le monde comme ceci plutôt que comme cela.

S'ils avaient été confrontés au postulat de cette toute puissance, définie par l'absence de contraintes, les Grecs, sans doute, auraient dénoncé la laideur de l'hubris, de l'orgueil qui excède toute limite, de la décision despotique qui tire sa gloire de son arbitraire. Je ne discuterai ici ni des manières diverses dont des philosophes -- et je pense bien sûr d'abord à Leibniz -- ont tenté de rendre au Dieu despote les vertus de la sagesse, ni non plus de l'épineuse question de savoir comment raconter l'histoire qui produit cette figure du pouvoir par rapport auquel la raison humaine a à se situer. Pour Pierre Duhem, physicien-philosophe, c'est la gloire propre du christianisme que d'avoir créé, contre les certitudes de la tradition, une distance dramatique entre vérités nécessaires et vérités de fait, qu'il est possible de nier sans contradiction. Pour d'autres[2], cette histoire est d'abord celle des villes où, dès la fin du Moyen-Âge, la différence entre le possible et l'impossible est affaire de volonté, de spéculation, d'esprit d'entreprise, rebelle à tout ce qui ferait coïncider par principe ce qui est et ce qui doit être. Quoi qu'il en soit, il me semble difficile de surestimer l'importance de ce fait : le Moyen-Âge a créé une nouvelle figure du scepticisme. Il ne s'agit plus d'une pensée minoritaire, acceptant le risque de l'exclusion ou de la marinalité, mais du résultat d'une contrainte imposée par le pouvoir lui-même, par l'Église condamnant comme erronée du point de vue de la foi tout usage de la raison qui limiterait l'absolue liberté de Dieu.

C'est à partir du lien posé a priori entre raison et production de fiction que je propose d'aborder l'invention pratique singulière d'un nouvel usage de la raison, usage centré autour de la question <<qui est l'auteur?>>.

\section{Fictions}

Je proposerai ici une <<définition $>>$ générale de cette entreprise singulière qu'on appelle $<<$ sciences modernes $>>$. Il ne s'agit pas d'une définition neutre, qui se proposerait le défi d'être également adéquate pour tout ce 
qui, aujourd'hui, porte le titre de science. Il s'agit d'une définition singularisante, au sens où sa signification dépend des problèmes qu'elle permet de poser, des distinctions qu'elle permet de susciter. Je dirai que, par définition, les sciences dites modernes donnent raison à Étienne Tempier et à Urbain VIII : les phénomènes que nous observons peuvent $<<$ être sauvés $>>$ de multiples manières, chaque manière renvoyant à un auteur humain, à ses projets, à ses convictions, à ses lubies.

De cette définition suit un premier type de genre littéraire, celui des textes qui, avançant une proposition innovante, font valoir que la raison est de leur côté, que l'interprétation proposée doit être reconnue comme rationnelle, parce qu'elle répond aux critères d'une saine méthode scientifique. Ce sont les textes des $<<$ savants fous $>>$. Le $<<$ savant fou $>>$ s'avance seul, le plus souvent armé de faits qui devraient logiquement lui valoir l'assentiment général. Il exige que l'on prenne au sérieux les faits, comme le recommandent les traités d'épistémologie. Il s'indigne, au nom des valeurs de la science, que sa proposition ne soit pas reconnue comme scientifique. Il méconnaît par là que s'il existe une valeur distinguant les auteurs scientifiques de tous les autres, c'est qu'un auteur scientifique ne peut se revendiquer d'aucune valeur pour exiger comme son dû l'intérêt de ses collègues. Cet intérêt, il doit inventer les moyens de le susciter. Il doit inventer les moyens de vaincre le scepticisme qui est toujours a priori la $<<$ réponse normale >>; il doit inventer les moyens de faire reconaître que sa fiction n'est pas seulement une fiction parmi d'autres.

$<<$ Qui est l'auteur $>>$ de la fiction que Galilée oppose, à propos du mouvement des corps, à la science traditionnelle? Car c'est bien d'une fiction qu'il s'agit, d'un monde où des boules parfaitement rondes mettent des milliers d'années à descendre une faible dénivellation le long de plans inclinés quasi-horizontaux, d'un monde où les boulets tirés par des canons dressés à la verticale se révèlent, dans le fut même du canon, suivre une course oblique, d'un monde où un corps traverse la Terre de part en part le long d'un tunnel creusé tout exprès. Un monde que, deux siècles plus tard, un train lancé à quelque $200,000 \mathrm{~km} / \mathrm{sec}$, parcourra pour permettre à ses passagers d'échanger des signaux lumineux avec des observateurs postés sur les talus.

On peut certes dire qu'il s'agit d'un monde abstrait, idéalisé, géométrisé. $\mathrm{L}^{\prime}<<$ auteur $>>$ serait alors l'abstraction. Mais l'on n'aura rien dit, car l'on aura simplement répété l'objection sceptique de Sagredo : ce n'est qu'un monde répondant à une définition élaborée dans l'abstrait. La question est plutôt de savoir ce qui a été abstrait, ce qui singularise cette fiction. Le monde fictif proposé par Galilée n'est pas seulement le monde que Galilée sait comment interroger, c'est un monde que nul autre que lui ne sait comment interroger. C'est un monde dont les catégories sont celles du dispositif expérimental qu'il a inventé. C'est en fait un monde concret au sens où ce monde permet d'accueillir la multitude des fictions rivales à 
propos des mouvements qui le composent et permet de faire la différence entre elles, de désigner celle des fictions rivales qui le représente de manière légitime. Galilée n'est pas seulement un écrivain, quoi qu'il est hautement significatif que son activité ait fait de lui un écrivain prodigieux. L'activité de l'écrivain, qui distribue les rôles, les arguments, les références est l'aboutissement de la création d'un monde devenu capable de témoigner en faveur de la fiction qu'il avance, capable de garantir que ce que Galilée dit n'est pas qu'une fiction.

La vitesse des corps graves galiléens n'est pas une notion abstraite, le produit d'une $<<$ manière abstraite de voir les choses $>>$. Abstraite, séparable des mobiles qu'elle qualifie, était bien plutôt la notion médiévale de vitesse : donnez-moi un moyen de mesurer l'espace et le temps, et vous pourrez oublier la différence entre la pierre qui tombe, l'oiseau qui vole, ou le cheval qui, épuisé, à bout de souffle, va bientôt s'abattre; je vous dirai leur vitesse. La vitesse des corps galiléens - -- la vitesse que, dirions-nous aujourd'hui, définit la dynamique classique -- est inséparable des mobiles qu'elle définit, elle appartient aux seuls corps galiléens, à ces corps définis par l'existence d'un dispositif expérimental permettant de soutenir, face à la multitude concrète des propositions rivales, que cette vitesse n'est pas seulement une manière parmi d'autres de définir le comportement de ce corps.

Le monde de Galilée apparaît comme <<abstrait>> parce que beaucoup de choses en ont été éliminées, dont le dispositif expérimental ne permet pas de définir les catégories. $L^{\prime}<<$ abstraction $>>$ scientifique traduit d'abord et avant tout que les victoires sur le scepticisme sont toujours locales et sélectives -- la pierre mais pas l'oiseau, les flux laminaires, mais pas les flux turbulents, le bilan pondéral d'une réaction, mais pas la catalyse, le germe mais pas le $<<$ terrain $>>$, etc. --, toujours relatives à l'invention d'un moyen de faire la différence entre fictions. C'est pourquoi la différence entre ce qui peut être objet de représentation scientifique et ce qui est censé <<échapper>> à la représentation n'est pas de l'ordre de ce qu'une théorie, philosophique ou autre, pourrait fonder. Fonder signifie toujours se référer à un critère qui prétend échapper à l'histoire pour en constituer la norme. Avant Galilée, qui aurait tenu pour <<représentable $>>$ la vitesse galiléenne, une vitesse instantanée avec laquelle un corps ne parcourt aucun espace en aucun temps? Qui croit pouvoir <<se représenter $>>$ la lumière, qui n'est ni onde ni particule, mais qui peut, selon les circonstances, répondre à la représentation soit d'une onde, soit d'une particule? Les sciences ne dépendent pas de la possibilité de représenter, elles inventent les possi- bilités de représenter, de constituer une fiction en tant que représentation légitime d'un phénomène. La <<représentation>> scientifique, ici, a un sens plus proche de celui qu'elle a en politique qu'en théorie de la connaissance. 
$\mathrm{L}^{\prime}<<$ abstraction $>>$ est la création d'un être concret, entrecroisement de références, capable de faire taire les rivaux de celui qui le conçoit. Sagredo s'est tu non pas parce qu'il aurait été impressionné par l'autorité subjective de Salviati, ni non plus parce qu'il aurait été mené par une quelconque pratique intersubjective de la discussion rationnelle à reconnaître le bien fondé de la définition proposée. Le dispositif expérimental a fait taire Sagredo, lui a interdit d'opposer une autre fiction à celle que propose Salviati, parce que c'était précisément là sa fonction: faire taire toutes les autres fictions. Et si, après trois siècles et demi, on enseigne encore les lois du mouvement galiléen et les dispositifs qui permettent de le mettre en scène - -- plans inclinés et pendules --, c'est que jusqu'ici aucune autre interprétation n'a réussi à défaire l'association inventée par Galilée entre le plan incliné et le comportement des corps graves.

Le scientifique qui crée une $<<$ représentation abstraite $>>$ du phénomène, s'il relève de ma définition de la science (cherchez qui cela élimine...) n'est jamais seul dans son laboratoire, à la manière d'un sujet isolable qui se $<<$ représenterait $>>$ un phénomène. Son laboratoire, comme ses textes, comme ses représentations sont peuplés de références à tous ceux qui peuvent les mettre en question. Comment Pasteur se représente-t-il un microbe? Comme l'écrit Bruno Latour, $<<$ ce nouvel être microscopique est à la fois anti-Liebig (les ferments sont vivants) et anti-Pouchet (ils ne naissent pas spontanément).[3]>> Nous avons aujourd'hui multiplié les modes d'intervention des microbes dans nos savoirs et nos pratiques, mais l'identité de ces microbes est toujours la somme de ce que des auteurs ont réussi à leur faire affirmer contre d'autres auteurs. La représen-/pp. 12-13/ tation scientifique n'a d'abstrait que l'apparence, elle est aussi concrète que le monde des discours et des pratiques au sein desquels elle intervient.

Faire taire le scepticisme non pas en invoquant une instance quelconque, méthodologique ou métaphysique, qui permettrait de $<<$ fonder $>>$ une proposition scientifique, mais en prévoyant les fictions rivales et en inventant le dispositif, ou l'usage du dispositif, qui pourra les démentir, telle est l'invention qui tout à la fois prolonge jusqu'à nous -- qui avons plus ou moins oublié le Dieu despote d'Étienne Tempier -- le scepticisme qui renvoie la raison à la fiction et ne cesse de le vaincre, par production toujours locale, sélective et limitée des moyens de faire la différence entre les fictions.

Bien sûr, Galilée prétend (discours platonicien oblige) que le dispositif expérimental est seulement là pour illustrer la vérité des faits, vérité rationnelle qu'en bon accoucheur il mènera Sagredo et Simplicio à reconnaître d'eux-mêmes dès lors qu'ils se seront libérés des illusions des sens ou de l'autorité indue de la tradition. Bien sûr, Lavoisier affirme, dans la Méthode de Nomenclature chimique (1787), que le chimiste doit se dépouiller de l'imagination qui le porte au-delà du vrai, vers la fiction, et de toutes les qualités qui feraient de lui un $<<$ auteur $>>$, afin de permettre à la 
nature de dicter la description adéquate. Il s'agit là de discours $<<$ méthodologiques $>>$, discours qui posent les principes au nom desquels devrait être établie l'asymétrie entre la fiction proposée et les fictions rivales. Le discours méthodologique identifie en quoi les fictions rivales sont reconnaissables à leurs erreurs, leurs préjugés, leur naïveté, bref, ne sont que des fictions. Mais tant que dure la controverse, ce discours méthodologique ne fait pas plus autorité que la proposition qu'il semble fonder. Après la controverse, si cette proposition l'a emporté, il fera histoire, il dira pourquoi il était normal et rationnel que le vainqueur ait vaincu, il fera reconnaître cette victoire pour simple produit de la rationalité scientifique puisque toutes les interprétations rivales auront été reconnues comme simples fictions.

Le discours méthodologique est le bulletin d'un type de victoire qui a la singularité de vouloir taire l'événement que constitue la victoire, le fait qu'une fiction a trouvé les moyens de faire la différence. Il tient sa plausibilité du fait que, dès lors qu'un scientifique individuel se sait partie prenante de cette pratique collective concrète qu'est une science, il doit concevoir sa proposition de manière telle qu'elle puisse résister à la controverse. Il ne s'agit pas alors de se $<<$ dépouiller $>>$, de se $<<$ purifier $>>$, mais d'intégrer dans sa propre démarche la perspective de la polémique où se décidera le caractère $<<$ scientifique $>>$ de la proposition. En d'autres termes, le scientifique est bien plutôt un stratège qu'un ascète. Et les textes scientifiques doivent être lus comme des oeuvres stratégiques, s'adressant à des adversaires potentiels ou déjà identifiés, prévenant et détournant leurs attaques, et non comme la description transparente d'une démarche $<<$ objective $>>$.

Qui est l'auteur? Cette question, sous une forme ou une autre, est celle que pose toute controverse scientifique. Elle s'y présente le plus souvent sous l'aspect d'une dichotomie: ou bien le scientifique peut convaincre ses lecteurs-collègues qu'il n'est pas <<auteur $>>$ de ce qu'il propose, qu'il s'est borné à prendre en compte ce qui s'est imposé à lui comme cela se serait imposé à n'importe qui dans les mêmes conditions d'expérimentation, d'observation ou de formalisation, ou bien il échoue, et se trouve désigné comme auteur volontaire, c'est-à-dire de mauvaise foi; ou involontaire, c'està-dire malheureux, de sa proposition. Dans le premier cas, sa proposition fera, dans une certaine mesure, $<<$ autorité $>>$, et pourra, le cas échéant, être reconnue comme $<<$ vraie $>>$ au sens scientifique du terme. Dans l'autre, elle sera rejetée en tant que $<<$ non scientifique $>>$, c'est-à-dire, en fait, en tant que fiction, située en-deçà des mises à l'épreuve qui distribuent le vrai et le faux en sciences.

On peut donc voir dans les sciences modernes l'invention d'une pratique originale d'attribution de la qualité d'auteur, jouant sur deux sens qu'elle oppose: l'auteur, en tant qu'individu animé d'intentions, de projets, d'ambitions, et l'auteur faisant autorité. Il s'agit non pas d'une naïveté, que 
pourrait critiquer les théoriciens contemporains de la littérature, par exemple, mais d'une règle du jeu et d'un impératif d'invention. Chaque scientifique se sait, et sait ses collègues, <<auteur $>>$ au premier sens du terme. Cela importe peu. Ce qui importe, c'est que ses collègues soient contraints à reconnaître qu'ils ne peuvent faire de cette qualité d'auteur un argument contre lui, qu'ils ne peuvent localiser la faille qui leur permettrait d'affirmer que celui qui prétend $<<$ avoir fait parler la nature $>>$ a de fait parlé à sa place.

La pratique des sciences modernes ne présuppose donc pas que le scientifique puisse se $<<$ purifier $>>$ de ce qui fait de lui un auteur. La question est de savoir si cette qualité d'auteur peut être $<<$ oubliée $>>$, si l'énoncé peut être détaché de celui qui l'a tenu et repris par d'autres dès lors qu'ils acceptent dans leur laboratoire le dispositif expérimental dont cet énoncé dit le sens. La question est de savoir si ceux qui acceptent cet énoncé peuvent, ensuite, se retrouver en position de rivaux indépendants, ou si le fait qu'ils acceptent fait d'eux des disciples soumis à l'unanimité d'une idée.

Un énoncé scientifique, s'il est finalement accepté, est tenu pour $<<$ objectif $>>$, ne parlant plus de celui qui l'a proposé, mais du phénomène en tant qu'il reste disponible pour d'autres fictions. Il est alors censé revoyer non à l'auteur mais à la $<<$ nature $>>$ en tant qu'autorité, comme si c'était bien la nature qui, selon les termes de Lavoisier, avait dicté sa $<<$ vérité>> Il est assez inutile de se demander si cette $<<$ vérité $>>$ doit être conçue comme $<<$ reflet $>>$ de la réalité, $<<$ représentation $>>$, ou $<<$ traduction $>>$. La $<<$ vérité>> au sens scientifique ne répond pas à un critère positif auquel on pourrait la reconnaître. Elle marque un jalon historique, la constatation que la fiction d'un auteur a réussi, sur fond de scepticisme a priori, à faire autorité.

\section{Histoire}

Autorité et auteur ont, on s'en souviendra, la même racine, et les pratiques médiévales, dites scolastiques, leur donnaient un sens solidaire. Les <<auteurs >> au sens médiéval sont ceux dont les textes font autorité, ceux qui peuvent être commentés mais non pas contredits. Ce qui ne signifie pas du tout une pratique de lecture soumise, bien au contraire. Ainsi, dans la Somme de Thomas d'Aquin, les auteurs sont appelés à témoigner sur une question déterminée sous forme de citations abstraites de leur contexte. Le jeu et l'enjeu est de les mettre d'accord en s'en tenant, le plus souvent, à la lettre de la citation, non de discuter du sens que lui prêtait l'auteur. En d'autres termes, l'auteur fait <<autorité>>, mais Thomas se fait juge et traite l'auteur-autorité en tant que témoin appelé à comparaître : il doit 
présupposer que le témoin a dit la vérité, et son jugement devra tenir compte de ce témoignage, mais c'est lui qui décide activement de la manière dont ce témoignage sera pris en compte.

De même, chaque scientifique sait que chaque phénomène étudié peut en général se laisser interpréter de manières multiples. Cela aussi importe peu. Ce qui importe, ce qui est l'enjeu de la différence entre fiction à vocation scientifique et proposition reconnue comme scientifique, est l'invention active des moyens de constituer le monde interrogé en témoin, garant de celui qui parle en son nom.

La différence entre pratique scolastique et pratique scientifique n'est donc pas aussi radicale qu'on aurait pu le penser. Thomas reconnaît que les $<<$ auteurs $>>$ font autorité, mais il se comporte comme s'il se savait libre de déterminer la manière dont ils doivent être pris en compte. Les scientifiques reconnaissent pour seule $<<$ autorité $>>$ la $<<$ nature $>>$, les phénomènes auxquels ils ont affaire, mais ils savent que la possibilité pour cette $<<$ autorité>> de faire autorité n'est pas donnée. C'est à eux de constituer la nature en autorité.

La grande différence tient à la liaison entre autorité et histoire. Les scolastiques tentent de mettre les auteurs -- philosophes païens, docteurs chrétiens et Auteur divin de la révélation -- d'accord. Leur ambition est de stabiliser, d'harmoniser l'histoire. En matière de sciences, réussir à constituer la nature en autorité et faire histoire sont synonymes. Le scientifique, en tant qu'auteur, s'adresse non à des lecteurs, mais à d'autres auteurs, il cherche non à créer une vérité terminale, mais à créer une différence dans le travail de ses <<auteurs-lecteurs $>>$. Et c'est en termes de différence, en termes de risques et de promesses d'histoires que constitue l'énoncé, que cet énoncé est évalué et mis à l'épreuve. Le scientifique ne cherche pas un accord stable; il cherche à ce que l'histoire soit contrainte par ce qu'il fait, il cherche à ce que l'histoire doive en passer par le témoignage des choses qu'il a réussi à susciter, par la différence entre fictions et vérité qu'il a réussi à créer.

Les sciences donnent souvent l'impression d'une entreprise $<<$ anhistorique $>>$. Si Beethoven était mort au berceau, ses symphonies n'auraient pas vu le jour, si Newton était mort à quinze ans, un autre, à sa place.... Cette différence renvoie en partie à la stabilité de certains problèmes, en l'occurrence à la régularité observable des mouvements célestes, aux ellipses empirico-mathématiques de Kepler dont la question était sans doute capable d'insister. Elle n'est pas aussi générale que l'on peut le penser. Ainsi, je crois pouvoir affirmer que si Carnot était mort au berceau, la thermodynamique ne serait pas ce qu'elle est. En tout état de cause, l'insistance plus ou moins stable des problèmes n'est pas suffisante 
pour expliquer l'allure anhistorique des sciences. Il faut ajouter que cette allure traduit aussi le caractère intensément historique de cette entreprise, la liaison entre vérité et histoire. Les scientifiques innovateurs ne sont pas soumis à une histoire qui définirait leurs degrés de liberté, ils prennent au contraire le risque de s'inscrire dans une histoire et de tenter de la transformer, mais de la transformer d'une manière telle que leurs collègues et ceux qui, après eux, diront l'histoire soient contraints de parler de leur invention comme d'une <<découverte $>>$ que d'autres auraient pu faire. L'histoire des sciences n'a pas pour acteurs des humains $<<$ au service de la vérité $>>$, si cette vérité doit se définir par des critères qui échappent à l'histoire, mais bien par des humains <<au service de l'histoire $>>$ qui ont pour problème l'histoire, et la vérité, ici, est ce qui réussit à faire histoire.

Étrange histoire, alors, qui associe sur des modes sans cesse renouvelés les arguments humains et les témoignages des choses. $<<$ Les mots et les choses $>>$, pourrait-on dire, à cette différence qu'il s'agit ici non pas de $<<$ représentations $>>$ au sens où cette histoire serait purement humaine et concernerait une $<<$ réalité>> définie comme muette, mais de la tentative -impliquant des moyens le plus souvent coûteux, mobilisant laboratoires, accélérateurs de particules, observatoires, enquêtes, collections, expéditions - -- d'inventer les modes selon lesquelles cette <<réalité>> se verra reconnaî-/pp. 17-18/ tre le pouvoir d'intervenir parmi les auteurs humains, de $<<$ faire la différence $>>$ entre leurs arguments.

L'histoire des sciences n'est donc pas seulement l'histoire des controverses qui décident de l'indécidable question <<qui est l'auteur? >>: le monde ou l'humain qui l'interroge. C'est également une histoire où s'inventent les réponses à un autre sens de la question. Car $<<$ qui est l'auteur? $>>$ peut aussi signifier $<<$ quelles passions et quels risques définiront l'auteur? >>, ou encore $<<$ de quel genre relèveront les fictions susceptibles de faire histoire?>>

Je proposerai de considérer qu'à la création de l'objet galiléen a correspondu l'invention de l'auteur Galilée, qu'à la création des sciences expérimentales correspond l'invention d'un type d'auteur qui ne préexistait pas à cette création, tout à la fois poète et juge; et parfois, également, prophète.

\section{Poètes et juges}

Qu'est-ce qu'un poète? Étymologiquement, c'est un fabriquant. L'histoire du langage a fait dériver le terme, mais a également dramatisé l'une des 
implications de l'art de la fabrication: la dimension de création qui ne se réfère à rien d'autre qu'à elle-même et n'a de compte à rendre à rien d'autre qu'à elle-même. Le poète, alors, est celui qui se donne la liberté, et en prend le risque, d'inventer, de faire exister, ce dont il parle.

Qu'est-ce qu'un juge? Au sens le plus général, c'est quelqu'un qui parle et agit $<<$ au nom de $>>$. Au nom du code, bien sûr, lorsqu'il s'agit de justice, mais il ne s'agit pas seulement de justice. Le juge existe dès lors que ce au nom de quoi il parle et agit l'autorise à déterminer ce qui, dans une situation concrète, est significatif, doit être pris en compte, et ce qui est secondaire, simple bruit parasite qui peut être $<<$ abstrait $>>$, éliminé effectivement ou intellectuellement. Le juge est celui qui sait, a priori, selon quelles catégories il convient d'interroger et de comprendre ce à quoi il a affaire.

La démarche expérimentale, telle qu'elle s'est inventée au 17ème siècle, fait donc de l'auteur scientifique un hybride singulier entre le juge et le poète. Mais le terme <<démarche>> est dangereux. C'est sous-entendre que l'expérimentation est un droit général, qu'elle peut s'appliquer partout de manière pertinente. Plutôt que de parler de démarche, il faut bien plutôt parler de l'événement que constitue, et que continue depuis à constituer, chaque fois qu'il se produit, la découverte pratique de la possibilité de soumettre un phénomène à l'expérimentation.

Soumettre un phénomène à l'expérimentation, c'est le mettre activement en scène, le $<<$ recréer $>>$, et pouvoir faire admettre que cette recréation n'est qu'une $<<$ purification $>>$, s'est bornée à éliminer des $<<$ effets parasites $>>$ de manière à rendre le phénomène capable de dire sa vérité. Le scientifiquepoète $<<$ crée $>>$ son objet, il $<<$ fabrique $>>$ une réalité qui n'existe pas telle quelle dans le monde mais qui est bien plutôt de l'ordre de la fiction. Le scientifique-juge réussit à faire admettre que sa création est une découverte, que la réalité qu'il a fabriquée témoigne de ce que les hypothèses au nom desquelles elle a été créée sont précisément celles qui rendent intelligible le phénomène $<<$ naturel $>>$ étudié. Le pouvoir créateur du poète doit se différencier du droit du plus fort, et depuis Étienne Tempier nous avons admis qu'aucun phénomène n'est naturellement $<<$ plus fort $>>$ que notre capacité à fictionner. La création expérimentale doit faire reconnaître qu'elle n'est rien d'autre qu'une purification légitime donnant au phénomène naturel le pouvoir de témoigner en faveur de l'expérimentateur. C'est pourquoi l'échec que redoute le plus l'expérimentateur est l'artefact, le $<<$ fait de l'art >> : il a bien $<<$ créé $>>$ une réalité expérimentale, mais cette réalité a été reconnue comme incapable de témoigner à propos du phénomène naturel. Il a créé, mais sa création n'a pu conquérir le titre de $<<$ simple purification $>>$; il a créé une fiction de laboratoire.

Le genre littéraire auquel correspond le mieux la littérature expérimentale est le genre épique ou initiatique: un /pp. 19-20/ <<héros >>, à travers des 
épreuves multiples au cours desquelles il se transforme lui-même, réussit à conquérir un trésor, un secret, une réponse. Qui est le héros? Ce n'est ni le scientifique ni le phénomène, c'est le lien qui les unit, c'est en quoi le phénomène interrogé rend le scientifique capable d'affirmer en son nom. Quelles sont les épreuves? Ce sont les questions, les objections, les possibilités de mise en controverse qui, au sein du texte, sont mises en scène plus ou moins explicitement et contrecarrées. Qu'est-ce qui se transforme? Ni le scientifique, ni le phénomène <<naturel $>>$ au sujet duquel la quête a été entreprise, mais le dispositif expérimental qui devient capable de donner sens, de son propre point de vue, à ce qui le menaçait, et de supprimer la menace ou mieux, de la reconvertir parfois en argument en faveur de son créateur. Le pire ennemi devient l'allié. Quel est l'objet de la quête? Le pouvoir de dire, face à des collègues que l'on suppose désormais réduits au silence, $<<$ la nature a parlé>> [4].

L'art de l'expérimentateur a partie liée avec le pouvoir: invention du pouvoir de conférer aux choses le pouvoir de conférer à l'expérimentateur le pouvoir de parler en leur nom. Le plus souvent l'événement que constitue la conquête de ce pouvoir se dissimule, dans les textes publics, derrière la description tranquille d'un ensemble d'expériences menant naturellement à une conclusion rationnelle. Mais, pour les lecteurs auxquels il s'adresse, ce texte est loin d'être $<<$ froid $>>$, c'est un dispositif risqué qui met en scène à la fois et indissociablement les $<<$ faits $>>$ et les lecteurs, et qui propose à ceux-ci des rôles -- critique pertinent, autorité incontestable, allié, rival malheureux - -- qu'ils auront à accepter ou à inventer les moyens de refuser.

En général, lorsqu'un fait expérimental est accepté, très souvent dans le processus même de son acceptation, une nouvelle question, une nouvelle histoire commence: de quoi témoigne ce fait ? Qui devra tenir compte de lui? Qui sera affecté par la contrainte nouvelle qu'il constitue? Bref, quelle est sa portée, c'est à dire de quelle histoire est-il porteur?

Parfois, cette question s'annonce avec une discrétion qui signale une grande confiance. Ainsi, à la fin de la lettre à Nature où ils annoncent la structure en double hélice de ce que nous appelons désormais l'ADN, Watson et Crick remarquent qu'<<il n'a pas échappé à notre attention que l'appariement spécifique que nous avons postulé suggère immédiatement un mécanisme possible de copie pour le matériel génétique >>. Pour tous ses lecteurs, cette petite phrase annonce, froidement, un événement bouleversant. Reconstituer, à partir de l'ensemble des données expérimentales disponibles, la structure d'une macromolécule que l'on trouve dans toutes les cellules était certes chose importante, et pouvait même valoir le prix Nobel. Mais la double hélice n'est pas une structure moléculaire comme les autres. Elle semble $<<$ parler d'elle-même $>>$, <<suggérer immédiatement $>>$ une solution au vieux problème des biologistes: comment, lorsque deux cellules se divisent, chacune peut-elle posséder la totalité de l'héritage génétique de son espèce? Et qui plus est, cette solution, 
une molécule capable de se copier elle-même, est de type technique, comme si le vivant avait, avant les hommes, découvert dans le texte que l'on peut copier, lettre à lettre, sans le comprendre, la réponse au problème de la transmission de la mémoire. Watson et Crick savent que la portée du $<<$ fait $>>$ qu'ils rapportent est inestimable, ils peuvent donc se contenter d'une $<<$ petite phrase $>>$ qui figure désormais dans l'histoire rhétorique des sciences comme l'exemple le plus accompli de litote.

Le plus souvent, pourtant, un $<<$ fait $>>$ n'est pas de lui-même aussi bavard. Sa portée, comme sa reconnaissance, renvoie à une histoire produit de stratégies actives. Qui <<intéressera>>-t-il? C'est-à-dire qui acceptera de lui lier son programme de recherche, de se laisser situer par lui, c'est-à-dire de le faire <<être entre>> (inter-esse) ses propres questions et celles qui l'ont produit? Question décisive, car d'elle dépend ce que nous devons appeler la création d'une réalité. Car la réalité, bien sûr, n'est pas ce qui existe indépendamment des hommes, mais ce qui démontre son existence à faire tenir ensemble une multiplicité d'intérêts et de pratiques disparates. Le Big Bang est-il une réalité? Si on peut jusqu'à maintenant hésiter à ce sujet, ce n'est pas parce qu'il est inobservable ou parce qu'il correspond à une singularité irreprésentable, mais bien parce que cette conséquence de la cosmologie relativiste n'a pas encore fait la différence pour d'autres sciences, n'a pas réussi à proposer à d'autres champs l'hypothèse de fictions qui pourraient les rendre solidaires. Les atomes, ou les microbes, ou le matériel génétique, existent-ils? Oui, affirment une foule de laboratoires académiques, médicaux, industriels, une foule de théoriciens divers, mais aussi de philosophes ou de spécialistes en matière éthique. Car s'ils n'existaient pas, nous n'existons pas non plus, nous qui avons été produits par une histoire que seule leur existence effective peut expliquer. Ce que nous disons $<<$ exister vraiment $>>$ a pour première caractéristique d'être la référence stable de pratiques changeantes. Mais cette stabilité elle-même est construite. Si un grand nombre de pratiques disparates ont réussi à se construire autour d'une référence commune, cette référence est stable par rapport à chacune d'entre elles.

L'histoire, dont nous sommes les héritiers lorsque, irrésistiblement, nous pensons que les microbes, le DNA, les atomes $<<$ existent vraiment $>>$, constituent les $<<$ causes $>>$ indélogeables d'une foule d'activités disparates, n'a pas pour seuls auteurs des juges-poètes. Elle est également le produit de fictions d'un autre genre, qui ont pour enjeu tout à la fois et indissolublement la question de la manière dont la réalité doit être comprise, et la question du rôle qu'une science particulière peut prétendre jouer dans cette compréhension.

\section{Grandes manoeuvres}


Avec la double hélice, la nature a parlé du matériel génétique, soit, mais a-telle dit que le matériel génétique constituait désormais la clef du vivant? A-telle dit aux embryologistes, par exemple, qu'il leur fallait reformuler leurs questions de manière à prendre le matériel génétique pour point de départ? Qu'il leur fallait comprendre le vivant qui se développe à partir de la bactérie, témoin fiable du rôle clef du matériel génétique précisément parce qu'elle ne se développe pas? $<<$ Oui! $>>$, ont affirmé ceux que l'on appelle les $<<$ biologistes moléculaires $>>$. $<<$ Non! $>>$, ont protesté des embryologistes scandalisés. $<<$ Peut-être bien $>>$, ont hésité les institutions de financement de la recherche, les jeunes chercheurs, les journalistes de vulgarisation, les rédacteurs de manuels scientifiques, tous ceux dont les choix, à ce niveau, construisent l'histoire. Une foule d'acteurs nouveaux pénètrent ici l'histoire. Les textes ne s'adressent plus seulement à des collègues, lecteurs-auteurs. Ils s'adressent à des lecteurs à qui est demandé de prendre en compte les conséquences d'une histoire qui n'est pas la leur, où ils ne sont pas auteurs. Corrélativement, le statut des auteurs change. L'auteur parle ici au nom de sa discipline. Ce sont la biologie moléculaire, la physique des hautes énergies, la science des neurones qui promettent, qui revendiquent, qui exigent.

Nous avons affaire à des textes que l'on pourra être tenté de lire selon la grille $<<$ vraie science $>>/<<$ idéologie $>>$. Lecture erronée si l'on entend par là que l'idéologie constitue une sorte de parasite, dont les sciences devraient ou pourraient se purifier. Lecture justifiée si celui qui dénonce $l^{\prime}<<$ idéologie >> se sait par là engagé dans une histoire qui le concerne parce que c'est une histoire qui a pour fonction non pas d'affirmer le triomphe local et sélectif sur le scepticisme, mais une réorganisation globale des savoirs et des pratiques humaines. Lecture ambiguè de toute façon, parce que la dénonciation se fait $<<$ au nom du droit $>>$ - -- droit à séparer le $<<$ vraiment scientifique $>>$ de ce qui ne l'est pas -- alors que ceux auxquels elle s'adresse sont en train de fabriquer le droit.

C'est que l'histoire qui se construit ici décide d'une modification, à plus ou moins grande échelle, du paysage des savoirs au sens le plus concret du terme: programmes de recherches, financement, prestige, recrutement des jeunes attirés vers les disciplines de pointe. Elle entreprend de réorganiser les questions, et ceux qui les posent, selon une hiérarchie dont elle constitue évidemment en général le sommet: questions essentielles, correspondant à la recherche fondamentale, questions hiérarchiquement secon- daires, $<<$ appliquées $>>$, questions parasites, obscurantistes, fausses, à abandonner, etc...

Les auteurs, ici, sont toujours juges, mais ils ne jugent plus les choses sans, en même temps, juger les savoirs et ceux qui les mettent en oeuvre. Ils sont toujours poètes, au sens de fabriquants, mais ils ne fabriquent pas seulement des liens entre les mots et les choses, des témoins fiables 
capables d'intervenir dans les discussions entre les hommes; ils fabriquent des concepts qui, au nom du lien créé, doivent opérer une transformation du paysage des savoirs, c'est-à-dire avant tout de la qualification des auteurs qui s'y activent.

Car le paysage des savoirs, à une époque donnée, qualifie les auteurs qui y opèrent. Certaines qualifications reviennent d'ailleurs à des définitions sociales. Ainsi, il est admis, depuis le début du 19ème siècle, que la science des ingénieurs se définit par l'application de théories physiques, et ne peut donc les mettre en question. Depuis longtemps, les ingénieurs connaissaient les phénomènes de non-linéarité qui passionnent désormais mathématiciens, physiciens et chimistes. Mais ils ne faisaient pas partie de ceux qui $<<$ font parler la nature $>>$... D'autres qualifications, en revanche, sont solidaires de prétentions disciplinaires plus précises. Ainsi, il y a huit ans, avec L'homme neuronal, Jean Pierre Changeux, au nom des sciences des neurones, a proposé que l'ensemble de ceux qui travaillent dans le domaine des sciences dites $<<$ de l'homme $>>$ soient qualifiés comme $<<$ manquant de $>>$, c'est-àdire $<<$ en attente de $>>$, cette $<<$ biologie moderne de l'esprit $>>$ que produira un jour les sciences des neurones. D'autres encore impliquent ce qu'on appelle une $<<$ conception du monde $>>$, c'est-à-dire une référence à la $<<$ vérité fondamentale >> de la nature en tant que principe de hiérarchisation des sciences. Ainsi, depuis le début du siècle, l'interprétation $<<$ probabiliste $>>$ de l'irréversibilité physico-chimique qualifie à la fois les phénomènes physico-chimiques et ceux qui les étudient: le véritable responsable de la différence entre ces phénomènes et la réalité que décrivent les lois fondamentales de la physique n'est pas la nature mais l'incapacité des humains à observer et à décrire ces phénomènes selon ces lois. Le physico-chimiste est alors jugé par son écart à l'idéal que constituerait le <<démon de Maxwell >> capable, lui, de décrire non des populations moyennes de particules, mais chaque particule individuellement.

Les textes qui se rapportent aux $<<$ grandes manoeuvres $>>$ où se modèle le paysage des sciences ne parlent pas de ce qui est, mais de ce qui sera, ou de ce qui devrait être. Ils n'annoncent pas l'événement que constitue une victoire locale et limitée par rapport à l'indécidabilité des fictions. Ils annoncent une fiction qui permettrait de juger en droit ce qu'ils n'ont pas les moyens d'atteindre en fait. Selon les cas, ils annoncent que ces moyens existeront un jour, ou bien qu'ils excèdent les capacités humaines mais permettent de qualifier ces capacités. Dans tous les cas, ces textes appartiennent à un genre prophétique, en se prononçant à propos de ce qui est $<<$ au nom $>>$ d'instances qui, d'une manière ou d'une autre, transcendent ce qui est. Et les controverses, ici, ne peuvent plus se régler dans les laboratoires, puisqu'elles ont pour enjeu la signification de ce qui s'y passe, le jugement que le laboratoire autorise à porter hors du laboratoire. C'est alors qu'apparaissent, plus ou moins explicitement, des arguments portant soit sur la rationalité scientifique, soit sur ce que nous a appris l'histoire des sciences; ou encore sur ce que nous pouvons et devons espérer pour l'homme, sur un plan économique, industriel, médical, 
historique, de tel type de développement, ou même sur la $<<$ vraie réalité $>>$ au-delà des apparences trompeuses.

Il faut insister encore sur le fait que le $<<$ genre prophétique $>>$ n'est pas un parasite de l'histoire des sciences. La <<réalité $>>$ technico-scientifique qui est le donné dont nous héritons a été produite par des juges-poètes-

prophètes. L'idéologie, en tant que catégorie désignant le passé, désigne des prophètes qui n'ont pas réussi à faire histoire, à faire de nous leurs héritiers. En tant que catégorie active au présent, elle désigne une controverse qui engage ceux qui y participent.

\section{Autres styles}

Juges, poètes et prophètes, ces termes désignent aussi bien des styles que des talents et des passions. Ces styles, talents et passions préexistaient bien sûr à l'invention des sciences, mais l'événement que constitue la découverte de la possibilité de sciences théorico-expérimentales les a constitués comme scientifiques: scientifiques, mais ne définissant pas pour autant les sciences.

La définition des sciences qui est mon point de départ est, je l'ai dit, singularisante. Elle engage une certaine manière de parler des sciences, et par exemple de poser le problème de ces textes que l'on peut dire scientifiques alors qu'ils n'empruntent à ceux que je viens de commenter que le style juridique. Les auteurs, ici, se font juges, sans pour autant être poètes, au nom de la méthode, de l'objectivité, ou du droit que toute science aurait de <<définir son objet $>>$. Ma définition m'engage à souligner qu'il s'agit de textes ennuyeux, où nul risque ne se prend, mais où les statistiques sont généralement impeccables car la mise à l'épreuve a pour cible la méthode.

Ma définition m'engage aussi à m'intéresser à l'ouverture de nouveaux types d'histoire, à la prolifération de liens nouveaux entre science et fiction qui compliquent la question <<qui est l'auteur?>> . Je veux parler, notamment, des simulations sur ordinateur qui transforment le statut de ce que l'on appelle modèle. Longtemps le $<<$ modèle $>>$ a signifié, dans les pratiques scientifiques, une activité $<<$ poétique $>>$ qui se sait incapable de faire de son auteur un juge. Le modèle a un auteur, qui ne peut prétendre se faire 
oublier. Mais le modèle simulé sur ordinateur introduit une telle distance entre les hypothèses de l'auteur et le comportement engendré que l'auteur parle de l'ordinateur comme l'expérimentateur parle du phénomène : comme de ce qui, convenablement interrogé, peut faire autorité. $<<\mathrm{La}$ simulation montre que... >> est un énoncé qui, désormais, tient parfois le rôle de $<<$ l'expérience montre que... $>>$. Ce sont là de nouvelles histoires qui commencent, avec de nouveaux types d'auteur, de nouveaux types d'enjeu et de nouveaux types de controverse.

Mais je voudrais ici parler d'une question par rapport à laquelle ma définition des sciences m'engage beaucoup plus activement, celle sans doute qui est en fait à l'origine de cette définition. Cette question est celle des limites du modèle expérimental qui suscite des poètes-juges-prophètes. S'il appartient à toute science (hormis les mathématiques et la logique, à laquelle le Dieu d'Étienne Tempier restait soumis) de tenter de faire intervenir la $<<$ nature $>>$ dans les arguments entre les hommes, rien ne dit que celle-ci doive toujours conférer le pouvoir du juge à celui qui cherche, activement, à la représenter. Toute expérimentation dépend de l'invention d'un rapport de force qui permet à la création de se dire simple purification, qui permet au poète de se dire juge. Mais ce rapport de force, il n'est pas au pouvoir des hommes de décider de le faire exister. La question qui m'intéresse est celle des styles de sciences qui peuvent s'inventer non pas, comme c'est le cas avec la simulation, à travers l'existence d'un nouveau dispositif technique, mais à travers l'invention positive, pratique, d'auteurs scientifiques qui s'adressent à la nature sans attendre d'elle qu'elle leur confère le pouvoir de juger.

Depuis quelques années, nous assistons non à la naissance mais à l'invention positive de ce que j'appellerai les $<<$ auteurs darwiniens $>>$. La distinction entre naissance et invention indique que c'est maintenant que Darwin est reconnu non seulement comme le fondateur de la science de l'évolution des espèces, mais comme le <<premier auteur darwinien $>>$, le créateur d'un style bien plus que d'une hypothèse explicative.

L'histoire de cette invention rétroactive comporte des épisodes très différents. Citons trois d'entre eux.

Le premier épisode est la résurgence de la controverse créationniste aux États Unis, le procès au cours duquel les darwiniens ont eu à faire valoir leur titre d'auteurs scientifiques face à des adversaires qui soulignaient la différence de leur pratique d'avec celle des auteurs juges et poètes: la $<<$ théorie darwinienne $>>$ ne donne pas les moyens de juger a priori ce qui, dans une situation, est important ou anecdotique; les <<concepts $>>$ d'adaptation, de survie du plus apte n'ont pas le pouvoir de rendre capable le scientifique d'anticiper la manière dont, dans telle situation, ils s'appliqueront. Le second épisode part de l'hypothèse due à Alvarez selon 
laquelle la disparition des dinosaures serait liée à la chute d'un météore géant, en passant par la mise en scène de $l^{\prime}<<$ hiver nucléaire $>>$, des effets similaires à ceux d'une chute de météore qu'entraînerait une guerre nucléaire, et continue aujourd'hui avec le problème de $l^{\prime}<<$ effet serre $>>$, qui met lui aussi en scène une dynamique d'effets centrés autour des échanges lumineux et calorifiques qui singularise la Terre. Le troisième épisode renvoie à la biologie évolutionniste proprement dite, et à la transformation de ses connections avec les données paléontologiques. La théorie des <<équilibres ponctués >> de Gould et Eldredge a mis en question le pouvoir de l'idée d'une sélection continue, graduelle, en conséquence de quoi les données paléontologiques, marquées par des discontinuités, devaient être jugées incomplètes et trompeuses. Corrélativement, la narration <<adaptationiste $>>$ a perdu le statut de narration unique; le mode narratif devient problématique, relatif notamment à la question de la stabilité ou de l'instabilité de l'identité spécifique.

De ces trois épisodes sont nés des auteurs dont le trait commun est d'avoir abandonné les risques du juge pour prendre ceux du narrateur (les modèles de l'hiver nucléaire et de l'<<effet de serre>> sont des modèles narratifs). On peut aller jusqu'à considérer que Wonderful Life[5] de Stephen J. Gould constitue un manifeste un peu analogue au Dialogue des deux grands systèmes $d u$ monde $e_{\llcorner}$non pas bien sûr parce que Gould serait un $<<$nouveau Galilée $>>$, mais dans la mesure où il s'agit de l'invention d'un nouveau style d'auteur caractérisé par un nouveau type d'<<ethos $>>$. Gould n'est pas un prophète au sens que j'ai défini; il ne parle pas au nom d'un pouvoir à faire reconnaître. Il communique le nouveau type de passion, de risque, d'intérêt qui singularise les auteurs darwiniens: l'invention des vivants et de la Terre en tant que témoins d'une histoire longue.

L'histoire des textes issus de la tradition darwinienne déçoit tous ceux qui attendraient d'une science qu'elle se fasse reconnaître à sa capacité de faire taire ceux qui la critiquent. Dès l'origine, elle a été mise au défi: comment une sélection aveugle pourra-t-elle jamais expliquer l'invention d'un organe aussi complexe que l'oeil! Et de fait, les <<évolutionnistes >> ne peuvent toujours pas nous raconter comment s'est créé un oeil, mais ils ont réussi à $<<$ faire histoire $>>$ avec les vivants d'une manière qui réinvente le regard que nous portons sur eux, regard qui a multiplié les questions, l'intérêt pour les traits curieux qui font percevoir ce que peut la durée longue là où, depuis Aristote, nous percevions la logique des rapports entre fins et moyens. Les $<<$ darwiniens $>>$ ont réussi à s'intéresser aux vivants, et à y intéresser beaucoup d'entre nous, sur un mode nouveau, à partir de la bizarrerie du $<<$ pouce du panda $>>$ [6], ou des tortues traversant l'Atlantique pour se reproduire, plutôt qu'à partir de l'adéquation de l'oeil à la vision. Ils ont réussi non pas à expliquer les vivants, mais à les constituer en témoins d'une histoire, à les comprendre comme racontant une histoire dont l'intérêt est qu'on ne sait pas a priori de quelle histoire il s'agit. 
Le genre darwinien s'apparente à celui du roman à intrigue: comment expliquer ce type de comportement, cette forme anatomique, ce mode de reproduction. Chacune de ces explications est locale: aucune ne confère à l'auteur le pouvoir de faire taire d'autres auteurs, qui s'occupent d'autres traits intrigants. Et pourtant les auteurs darwiniens ont réussi à $<<$ faire histoire ensemble >> c'est-à-dire à faire intervenir le témoignage d'un terrain dans la description des autres. Ce qui fait histoire, ici, se marque à la singularisation des histoires. Les auteurs darwiniens apprennent les uns des autres la vigilance, la nécessité d'explorer la diversité des causes et la diversité des manières, une même cause pouvant causer la méfiance par rapport à toute cause qui porterait avec elle la prétention de déterminer comment elle cause; bref, la méfiance par rapport à ce qui, corrélativement, s'identifie comme piège: les divers modes d'assimilation de l'histoire à un progrès. Dans Wonderful Life ${ }_{\llcorner} /$pp. $29-30 /<<$ le rôle $>>$ de Simplicio est tenu par $<<$ nos habitudes de pensée $>>$ qui toujours tendent à définir ce qui est arrivé comme ce qui devait arriver.

Les auteurs darwiniens ne sont donc ni juges, ni poètes, ni prophètes, parce que l'histoire de la vie telle qu'ils ont appris à la lire n'autorise pas de principes d'économie, c'est-à-dire ne permet pas d'inventer le rapport de force qui permettrait de juger, de créer un objet, de hiérarchiser les questions. Mais les auteurs darwiniens s'appuient néanmoins sur un rapport de force. Leurs questions présupposent et impliquent la stabilité de la différence entre le présent qu'ils interrogent, et le passé qu'ils tentent de raconter. Cette différence trouve son analogue dans les genres de la fiction: le propre du roman policier de facture classique, par exemple, est que la différence entre l'enquêteur et les suspects est stable. Le crime, s'il a eu lieu, a eu lieu avant l'intervention de l'enquêteur. La règle du genre, dans les récits de type darwinien, est du même type : les traits qui les intéressent sont le produit d'une histoire longue et ont donc une identité stable par rapport au type d'intervention qui permet de les étudier.

Tout autre est la situation de l'auteur scientifique lorsque ceux à qui il a affaire -- rats, babouins ou humains -- sont susceptibles de s'intéresser aux questions qui leur sont posées, c'est-à-dire d'interpréter de leur propre point de vue le sens du dispositif qui les interroge, ou encore de se mettre à exister sur un mode qui intègre activement la question. Il n'en va pas de même lorsque l'histoire, par laquelle celui qui interroge cherche à devenir auteur, fait également histoire pour celui qu'il interroge, c'est-à-dire lorsque les conditions de production de connaissance de l'un sont inévitablement des conditions de production d'existence pour l'autre. La notion de témoin, ici, devient ambiguè: avec les humains notamment, le scientifique a affaire à des êtres qui sont susceptibles de lui obéir, de chercher à le satisfaire, d'accepter, au nom de la science, de répondre à des questions sans intérêt comme si elles étaient pertinentes, voire même de se laisser persuader qu'elles le sont puisque le scientifique $<<$ sait mieux $>>$. 
Faut-il, pour souligner qu'ici science et éthique sont unis de manière indissoluble, rappeler l'expérience où Stanley Milgram créa, au nom de la science psychologique, les conditions où des individus normaux allaient devenir des bourreaux? Faut-il également, pour rappeler que la question éthique, ici, est toujours en même temps une question technique, souligner que l'expérience dite de Milgram n'a pas produit de témoins fiables? Elle n'a conféré aucune autorité à un énoncé particulier mais a bien plutôt reproduit, dans un cadre expérimental, la perplexité à laquelle nous contraint l'histoire. Les sujets-bourreaux de Milgram se savaient au service de la science, et ce savoir a pour conséquence que l'expérience, qui était censée se borner à mettre en évidence un comportement, a sans doute contribué, sur un mode incontrôlable, à le produire. Si un vivant est capable d'apprendre, c'est-à-dire aussi de se définir par rapport à une situation, le protocole qui vise à le constituer en témoin fiable sur le mode expérimental, à le contraindre à répondre de manière univoque à une question décidée par l'expérimentateur, crée un artefact.

Lorsque ce qui est interrogé ne peut être défini comme indifférent à la manière dont il est interrogé, lorsqu'il s'apprend, c'est-à-dire devient, selon la manière dont on l'interroge, l'auteur scientifique lui-même est exposé à de nouveaux types de risques, des risques que j'appellerais $<<$ pathétiques $>>$. En effet, dans la mesure où ce à quoi il a affaire ne peut plus faire $<<$ autorité $>>$, lui-même ne peut plus faire oublier, ou oublier, qu'il est partie prenante de ce qu'il décrit. Le scientifique est celui (ou celle) qui prend l'initiative de poser une question. Le $<<$ risque pathétique $>>$ est le risque de subir les contre-coups de son initiative, la question, en retour, du $<<$ qui suis-je pour l'autre? $>>$. Ces contre-coups peuvent être immédiatement physiques, comme ce fut le cas de cet éthologiste qui un jour se fit attaquer par un chamois: il avait cru <<observer sans intervenir $>>$, mais du point de vue des chamois, sa neutralité avait signifié $<<$ domination $>>$. Sans le savoir, il avait triomphé d'une série de défis visuels et était donc le $<<$ mâle dominant $>>$, jusqu'au jour où un rival choisit de passer au stade suivant de la confrontation... Le contre-coup peut être affectif: l'éthologiste Shirley Strum[7], minoritaire parmi ses collègues, nous a communiqué sa découverte, à savoir que comprendre les babouins, cela avait été aussi et en même temps prendre le risque de les aimer, c'est-àdire d'être transformée par eux. Le contre-coup peut mettre en question la qualité même du titre d'auteur scientifique. Comment étudier un groupe humain différent sans risquer sa propre identité sociale et culturelle, c'est une question à laquelle ont toujours eu à répondre les ethnologues, qui parlent de la tentation du $<<$ go native $>>$, de connaître un devenir qui vide de son sens le projet de $<<$ rapporter $>>$, à ceux qui ne sont plus dès lors des $<<$ collègues $>>$, ce qui a été appris.

Ces $<<$ risques du métier $>>$ définissent la singularité d'une $<<$ matière $>>$ qui n'a pas encore véritablement conquis la reconnaissance de son $<<$ genre littéraire $>>$. Ils sont le plus souvent aujourd'hui des états d'âme douloureux, soigneusement tus au nom de la méthode, sujets d'angoisse ou de 
dénonciation. Ils désignent pourtant, en négatif, le style d'auteur que cette $<<$ matière $>>$ pourrait susciter si un jour elle devient effectivement $<<$ matière de science $>>$, selon la définition que j'ai donnée de la science. Verrons-nous des auteurs scientifiques tenter de $<<$ faire parler $>>$ une réalité dont ils savent qu'elle est engagée avec eux dans un devenir commun? Verrons-nous des auteurs apprenant les uns des autres les risques de fictions qui rendent toujours plus discutables le sens et les implications de leurs propres initiatives? Il faudrait, dira-t-on, une passion $<<$ lucide>> que les scientifiques sont loin de posséder. Mais la passion de faire de la physique est, elle aussi, singulière, et celle des narrateurs darwiniens ne l'est pas moins. Il n'y a pas de science sans fiction, et il n'y a pas de fiction sans passion. Il n'y a pas de $<<$ passion scientifique $>>$, mais l'invention, toujours singulière, d'un $<<$ devenir scientifique $>>$ des passions.

\section{Isabelle Stengers}

Université Libre de Bruxelles

Surface Page d'Acceuil/Home Page

[1]Cité in P. Duhem, Sozein ta phainomena. Essai sur la notion de théorie physique de Platon à Galilée, Paris, Vrin, réédité en 1982, p. 134.

[2]Voir notamment E. Alliez, Les temps capitaux. 1. Récits de la conquête du temps, Paris, Les Editions du Cerf, 1991.

[3]B. Latour, <<D'où viennent les microbes $>>$, in Les Cahiers de Science et Vie. Les grandes controverses scientifiques ndeg.4, Pasteur. La tumultueuse naissance de la biologie moderne, août 1991, p. 47.

[4]Voir, à propos de ce tout ceci, Bruno Latour, La science en action, Paris, La Découverte, 1989.

[5]Paru en français sous le titre La vie est belle, Paris, Le Seuil, 1991.

[6]Titre d'un livre de Stephen J. Gould paru chez Grasset en 1982. Voir aussi Le sourire du flamant rose, Le Seuil, 1988, ainsi que Quand les poules auront des dents, Fayard, 1984.

[7]S. Strum, Presque humain. Voyage chez les babouins, Paris, Eshel, 1990. 